\title{
I linguaggi del dialetto: il fonoritmo di Achille Curcio
}

\author{
Luigi Tassoni \\ Università di Pécs
}

A Milly

I lettori della poesia dialettale italiana, che così indicativi esempi ha mostrato alla nostra contemporaneità, si trovano naturalmente in una duplice posizione. Da un lato percepiscono come motivata la scelta topica della propria lingua, sia pure a circolazione limitata presso una cerchia di parlanti, dall'altro scoprono la specificità del linguaggio del testo che apre ad una condizione d'ascolto accessibile anche al di là della cerchia dialettofona; da un lato si trovano davanti ad una parola sopravvissuta alla periferia dell'impero dei linguaggi della comunicazione internazionale, dall'altro scoprono la sopravvivenza di un modo, un senso, un carattere, un'intonazione propria musicale tipica della specifica dialettalità; da un lato, questi lettori, si sentono prescelti fra pochi all'ascolto del testo «in una lingua che più non si sa», dall'altro testimoniano che il loro «piccolo» dialetto (a confronto dei grandi numeri delle "grandi» lingue) tocca un nodo ancestrale tipico per appartenenza, familiarità, vicinanza. Ovvero i lettori della poesia dialettale di oggi constatano l'importanza del passaggio dall'oralità alla scrittura della poesia, che mette fortemente in rilievo il dato fondante di ogni testo che è la sua vocalità, la parte del significante fonico e ritmico.

La poesia dei dialetti italiani riflette l'attuale condizione della letteratura che agisce tramite il potere incantatorio del verso, in un'epoca in cui la necessità della comunicazione sembrerebbe aver dimenticato la funzione principale del significante fonico e ritmico che imbastisce il messaggio prima ancora che ce ne siamo fatta una ragione logicamente significata, e anzi a volte introducendo a percorsi del Senso imprevisti dal discorso in sé. Come ricorda Leopardi in un pensiero dello Zibaldone dell' 8 gennaio 1820: «E' cosa osservata dagli antichi poeti ed artefici, massimamente greci, che solevano lasciar da pensare allo spettatore o uditore più di quello ch'esprimessero».

Ora, un tale effetto riguarda regioni come la Calabria che per ventura e fortuna storica sono attraversate ancora e fortemente da una molteplicità di dialetti, sia pure per aree di somiglianze. 
Quando Gerhard Rohlfs viaggiava in Calabria per aggiornare la sua Grammatica storica della lingua italiana e dei suoi dialetti, gustava con piacere questa prolificità di differenze dei dialetti calabresi (che, sia detto per buona pace del dialettologo, sono raggruppati per isotopie riconoscibili). E quando Rohlfs si fermava sulla costa del mare Jonio, dove la dolce collina degrada verso il Golfo di Squillace nel catanzarese e prima dell'Aspromonte, incontrava un poeta, Achille Curcio, che lo aiutava a saggiare il polso della situazione. Achille Curcio scrive da più di mezzo secolo in un dialetto che si è mosso fra le radici della lingua appresa tra Montauro e Borgia, le cittadine della propria infanzia, e sviluppato come lingua della poesia, anche con i più recenti recuperi di una dizione arcaica, nel senso del riuso (o riesumazione come per la parola temetum di cui parla Agostino nel De Trinitate: un significante del quale s'era persa la corrispondenza con il referente), contestualizzato e risignificato nella magia del testo.

Il libro più recente del poeta settantacinquenne di fatto si distingue dai cinque che lo precedono per la scelta di far funzionare come forza del codice il significante arcaico entro la riconoscibilità della struttura odierna del dialetto. In questo sesto libro, 'U poeta non rida (2005) (il poeta non ride), la caratteristica comune al resto dell'opera, è l'invenzione di un flusso fonoritmico sulla base della metrica moderna. Questo speciale fonoritmo, che fa la vocalità del testo e porta al discorso nuovo Senso, vogliamo illustrare con il necessario esercizio del commento ai testi.

La poesia eponima del volume di cui stiamo parlando, 'U poeta non rida, presenta una situazione tipica della dialettalità di Achille Curcio sia dal punto di vista formale del testo sia da quello tematico. Ovvero dialettalità come linguaggio per la storia dei sopravvissuti, il margine estremo e non realistico dal quale l'io dà senso alla storia e guarda al mondo con quell'inquietudine del tutto irrisolto, della precarietà, dell'insanabile frattura fra desiderio ed evento, infanzia e storia. Il pianto (di cui parla al v.2), piuttosto che essere letteralmente disperazione per una perdita, è invece, come il lamento e il llanto ispanico, constatazione, accertamento, testimonianza, affermazione della perdita irreversibile. Sotto quale luce si collocherà il tema della memoria, il lettore lo intuisce da sé.

Tutto è davvero perduto: lo sguardo dell'io come quello della luna stanno fuori dal tempo. Per Leopardi solo la luna è l'agente testimone magico sottratto alla legge che consuma il tempo, e per Curcio la testimone esterna, sottratta al tempo, ha una diretta corrispondenza con l'io, con la voce che parla nel testo, con la figura del poeta (sottratto, in questo momento, alle leggi del tempo), mentre il tempo umano distruttore entra inevitabilmente nelle case, striscia letteralmente fra le cose. La conclusione riguarda la perdita irrecuperabile, nel tempo e nella storia, e la visibilità della deriva umana, senza possibilità di risanamento in questa specialissima coscienza diretta della differenza e della comparazione. Il senso generico che traiamo dalla lettura del testo è in effetti articolato e non così sommario come può apparire nella nostra parafrasi, è necessario dunque seguirne il senso di strofa in strofa, leggendo 'U poeta non rida in modo da riconoscere la funzione costruttiva del discorso assunta dalle strategie formali del testo (mi è impervio il senso): 
'U poeta non rida:

guarda e ciangia

si ncuntra nu guagliuna gialinusu,

chi va stendendu 'a manu virgugnusu

mu ricogghia nu signu, na carizza. (vv.1-5) ${ }^{1}$

L'avvio del testo è in effetti (vv.1-2) introdotto da un endecasillabo spezzato, e produce richiamo fonico (ridalguarda), e rima tematica (vv.3-4), con gli attributi (gialinusu/virgugnusu) del ragazzo che stende la mano, e il v. 5 in rima con il verso successivo (carizzalamarizza).

$\mathrm{Cu}$ nu cora mpuciutu d'amarizza

ciangia e guarda

facci senza culura, arrepicchiati

comu vecchi damaschi,

de mammi chi nt'o chiantu

strudiru l'occhi stanchi,

perciati de nu lampu de lupara. (vv.6-12)

Nel periodo strofico si evita la rima in cerca di risonanze interne (cora, v.6, culura, v.8, lupara, v.12), e si inverte la coppia verbale del quadrisillabo (ciangia e guarda, v.7), che descrive gli incontri spettrali (facci senza culura, v.8) con il riferimento alle madri che consumano gli occhi trafitti da un colpo di lupara. In questa strofa si disegna esplicitamente il campo semico della consumazione (i visi gialli e senza colore, la pelle raggrinzita come un vecchio damasco: con allusione alla nobiltà della stoffa). Fra l'altro l'immagine degli occhi delle madri perciati dal colpo di lupara sottintende l'immagine logicamente deducibile dei figli uccisi dal colpo di lupara, con un uso dunque metanarrativo dell'ipallage, che cancella la costruzione logica del racconto in modo da farne apparire un'immagine sovrapposta e derivata: gli occhi delle madri consumati e feriti come le ferite mortali sul corpo dei figli.

'U poeta non rida e resta sulu mu guarda e pemmu senta 'u tempu, chi leggeru trasa pe ogni casa, striscia supra ogni cosa e, senza 'u ti nd'adduni, t’arrobba nu suspiru, na jornata. (vv.13-19) $)^{3}$

1. Nelle note, per comodità del lettore, trascrivo la versione italiana dei testi tradotti dal poeta. «Il poeta non ride: guarda e piange se incontra un bambino pallido, che timido stende la mano per raccogliere un segno, una carezza.»

2. «Col cuore carico d'amarezza piange e guarda visi pallidi, aggrinziti come vecchi damaschi, di mamme che nel pianto hanno consumato gli occhi, trafitti da un lampo di lupara».

3. «Il poeta non ride e resta solo a guardare e percepire il tempo, che leggero entra per ogni casa, striscia sopra ogni cosa e furtivamente ti ruba un sospiro, un giorno della tua esistenza». 
La strofa riprende l'incipit e la rima interna della prima strofa e introduce al tema del tempo inseguito perché l'io lo percepisca: leggero il tempo entra nelle case, striscia sopra le cose, e ruba furtivamente nu suspiru, na jornata (v. 19). Anche qui si evitano le rime consonantiche a fine verso a favore di assonanze o risonanze e rime interne: poeta (v. 13), resta (v. 13), senta (v. 14), jornata (v. 19); trasa (v. 16), casa (v. 16), cosa (v. 17); leggeru (v. 15), suspiru (v. 19), nel cerchio chiuso dei due endecasillabi, ad apertura e chiusura di strofa, con settenario evidenziato in cesura, che racchiudono quattro settenari.

Notiamo come la difformità dell'alternanza metrica, diversa di strofa in strofa, costituisca il fondo di una dizione che raggiunge la significazione per echi. La rarità della rima consonantica e $\mathrm{i}$ "nodi» dei richiami fonici costituiscono il flusso attraente di un discorso regolarmente asimmetrico, dove la mancanza di corrispondenze regolari sul piano formale introduce alla mancanza di corrispondenza tra l'io e il mondo ('U poeta non rida e resta sulu, v.13).

'U poeta capiscia ca nt'o mundu

'a vita è sulu sonnu:

nu sonnu a voti longu,

e a voti curtu e amaru

che poi diventa eternu. (vv.20-24) ${ }^{4}$

La breve strofa formata da un endecasillabo $(7+4)$ e quattro settenari riprende il soggetto ('U poeta capiscia), e si svolge per ripetizioni ad eco (sonnu/sonnu, vv. 21-22), comparative (a voti / a voti, vv. 22-23), foniche (poetalvitalvoti/diven$t a$, vv. 20-24), fino alla ripetizione che dà il la alla strofa successiva. Qui il tema è il sogno (nel dialetto del catanzarese sonnu designa sia sonno che sogno), ovvero il desiderio continuo che può essere lungo o corto e amaro, e che per tutti arriva all'eternità. $\mathrm{Al}$ "poi» della morte della strofa si associa il "poi» della vita, della fine della giornata, nella successiva:

Poi 'a sira, quandu 'a genta

si jetta, scamazzata de fatica,

mu procura 'e riposu nu tornisa,

'u poeta s'affaccia a la finestra

e vida ca nt'o celu

cumpara ancora 'a luna,

'a stessa luna antica,

vecchia guardiana amica

de tutta sta miseria. (vv. 25-33)

4. «Il poeta comprende che sulla terra la vita è soltanto un sogno: un sogno a volte lungo a volte breve e amaro e che diventa eterno».

5. «A sera poi, quando la gente crolla, schiacciata dalla fatica per procurarsi un po' di riposo, il poeta si affaccia alla finestra e scorge che in cielo compare ancora la luna, la stessa antica luna, vecchia e amica sentinella di tutta questa miseria». 
L'interessante struttura metrica propone in apertura un settenario (con il vocalismo delle due sinalefe), più tre endecasillabi seguiti da altri quattro settenari, con le uniche rime consonantiche ai vv. 26, 31, 32, e il consueto continuum di risonanze foniche interne, là dove si determina il passaggio dall'immagine del poeta che guarda a quella del poeta che vede, affacciato alla finestra (spettatore muto), e vede il proprio referente «fuori dalle leggi del tempo», ovvero la luna amica e antica guardiana (che sta a guardia e guarda), perfezionando così il precedente pensiero sul sonno eterno che rimane desiderio per l'umanità, e raffigurandosi l'immutabilità e l'affidabilità dell'agente esterno che è la luna. Fuori di retorica, questa miseria, su cui chiude la strofa, della quale sta a guardia la luna, è proprio la miseria dell'umanità, la sua condizione elementare di rinuncia al sogno e al desiderio.
All'arva supra i hjuri
poi trova l'acquazzina:
lacrimi de sta luna
chi, guardando sta terra,
addhucia 'a notta e ciangia:
comu fannu i poeti pe campara. (vv.34-39)

Si noti il passaggio temporale dal momento della sera all'ora dell'alba del nuovo giorno: la nuova immagine (settenari con endecasillabo in clausola) è quella della rugiada, acquazzina, che è fatta di lacrime lunari posate su fiori per addolcire la notte, che per noi si prolunga nell'immagine del canto del poeta che, "posato", addolcisce la vita. La metafora, prolungata nella contemporaneità sul calco della tradizione secentesca, in effetti risponde al perché dell'immagine forte e paradigmatica del poeta che non ride. Spieghiamocela così: il poeta non ride, e se piange il suo è pianto-canto, il fonoritmo della poesia, il pianto-canto di un testimone lunare, materialmente modulato, un piantocanto «senza parole», o che fluisce al di là delle parole.

Semplice e intrigante, la posizione della voce nel testo aspetta la richiesta di una parola dolce, come dice in un altro testo Achille Curcio (Cercami quandu $v o i)$ : "Cercami quandu voi / ncuna parola ducia; / cercami quandu voi,/ aspettu pemmu sentu na chiamata,/ na vuciata d'aiutu». ${ }^{7}$ Parola dolce o canto modulato nelle forme che qui abbiamo emblematicamente riassunto, o discorso senza-parole, discorso che supera l'in sé della parola, e discorso sulla miseria, sul deserto, sulla solitudine, sulla delusione, sull'abbandono, di un sopravvissuto al cataclisma. Ecco, la dialettalità di Achille Curcio è quella del sopravvissuto al cataclisma, di colui che sull'orlo del vulcano, nel paesaggio della ginestra fiore del deserto, comunque resiste come resiste la sua lingua modulata sottovoce nell'immensa circolazione dei potenti linguaggi della contemporaneità.

6. «All'alba poi, sopra i fiori, ritrova la rugiada: lacrime di questa luna che, guardando la terra, illumina e piange: come fanno i poeti per campare».

7. «Cercami quando desideri una parola dolce; cercami come vuoi, rimango ad aspettare un'invocazione d'aiuto". 
Ancora un testo significativo del volume 'U poeta non rida testimonia questo aspetto elementare ed essenziale del poeta dialettale italiano nella nostra epoca. Si tratta di Na crozza supra mara, che forse riecheggia e trasforma, ma con una visionarietà notturna, il semplice motivo della canzone popolare Vitti na crozza sutta lu cannuni:

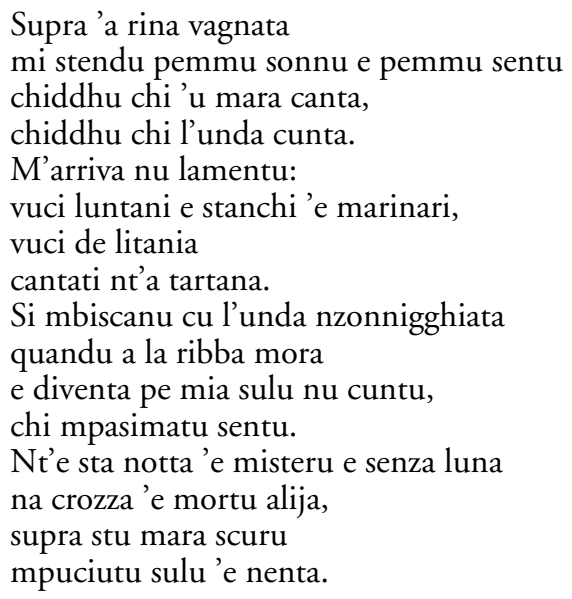

Sono le rime assonanti tematiche e le ripetizioni a svolgere il discorso letteralmente (al solito endecasillabi e settenari ne regolano il passo): sentu (v. 2), canta (v. 3), cunta (v. 4), lamentu (v. 5), cuntu (v. 11), sentu (v. 12), nenta (v. 16). Associate alle ripetizioni interne (chiddhu chil chiddhu chi, vv. 3-4; vuvi/vuci, vv. 6-7), e alle diversificazioni semiche (litania, v. 7, cantati, v. 8: si noti che il testo dice che le voci sono cantate, e indirettamente, in quanto voci di litania) designano il campo semico del cantare. Qui il canto è associato a quello del cunto, racconto, ma un racconto del nonsenso, del niente, della visione mortale nullificante. Questo cantu-cuntu era il motivo conduttore del libro Chi canti, chi cunti? (1983), ben riassunto nella poesia eponima, dove si trovava già il motivo del mare che canta e racconta (e anche incanta): "O mara de sempa, /chi canti, chi cunti/ cu st'undi sbattendu,/ cu 'a schiuma vasandu/ sta rina lucenta,/ sta praia apparata?» (vv. 1-6). ${ }^{8}$

Ora scopriamo l'io in posizione d'ascolto, letteralmente sdraiato sulla sabbia bagnata, per ascoltare il cantu-cuntu del mare, omologo e parallelo al cantoracconto del testo. Il cuntu è dapprima il lamento e la litania come voce di marinai, voce che si mescola all'onda nzonnigghiata, assonnata e dunque lenta, lieve. L'io ascolta (sentu) essendo mpasimatu, pieno di stupore e anche con lo spasmo dell'ascolto in sé. L'attributo, arcaico, è effettivamente un ripescaggio nella memoria dialettale che ristabilisce come valore attuale, con la suggestio-

8. «O mare di sempre, cosa canti, che cosa racconti sbattendo con le onde e baciando con la tua schiuma la rena spianata?». 
ne del significante fonico, il significato perduto del termine (è l'effetto temetum, già citato, di cui parla Agostino). Proprio questo risuscitare da morte la parola della memoria corrisponde sul piano del discorso all'apparizione della croz$z a$ di morto, il teschio della canzone popolare, che alija, cioè manda letteralmente un alito. Ecco qui aggiunto ancora un elemento al tema e al campo semico della parola-canto-racconto: il canto del mare, il racconto dell'onda, il lamento, la voce, la litania dei marinai, il rumore dell'onda assonnata, il sospiro della inquietante crozza di morto. Si raggiunge praticamente per sinestesia l'effetto di annullamento, di nullificazione dei versi finali, là dove si associa l'affievolirsi dell'ascolto alla visione del mare scuro, ovvero alla sparizione del visibile. Il mare scuro è mpuciutu sulu 'e nenta, cioè per ossimoro riempito o pieno di niente.

In questo testo, dunque, l'ascolto del niente si riferisce alla visione del niente, al canto del niente, che pone in primo piano la stessa autonomia e autosufficienza del canto, del canto in sé, della risonanza, e del discorso della poesia che si spinge fino al suo limite estremo, consapevole di potere e dover parlare del niente. Potremmo assumere questa come condizione emblematica della dialettalità in poesia, la condizione nella quale si elabora un linguaggio intorno all'impossibilità dell'ascolto, e contemporaneamente quel linguaggio conclama prima di tutto la sopravvivenza della lingua, la sua sopravvivenza nel testo. Al lettore spetta il difficile ruolo di scongiurarne la cancellazione.

Il canto esiste, la litania, le voci, il racconto, in esso consistono, e ciò perché e se, malgrado tutto, il lettore è capace di «sentire» il testo. Le strategie di formazione del Senso nella poesia di Achille Curcio privilegiano lo spazio del significante fonoritmico, che domina come portatore di un cantabile la struttura del testo, la sua seducente attrazione per l'orecchio, che si puntella comunque su un discorso (lo abbiamo visto) articolato fino a far apparire naturalmente dicibili e comprensibili le figure retoriche che invece sottolineano l'illogico, l'irrapresentabile, il controsenso della storia come del discorso poetico. La serrata comparazione per sinestesia, la cruda forza dell'ossimoro, la sinteticità drammatica dell'ipallage, l'apertura della metafora, testimoniano altrettanto bene come il testo poetico, per Curcio, sia un luogo del dire come differenza, del mostrare come definitiva assenza, e soprattutto della lingua come confronto comparativo.

Il poeta cioè mostra dalla parte del testo la posizione e il ruolo che per sua natura il lettore di poesia dialettale può assumere nella nostra contemporaneità. Lettore che sa passare dal comparativismo spontaneo al comparativismo consapevole, che in fondo gli dà conferma della propria operazione di confronti relazionali per la consentaneità dei codici linguistici e della scrittura poetica. Non posso non ricordare che Claudio Guillén, maestro di comparativisimo, nel suo famoso studio intitolato nella versione italiana L'uno e il molteplice segnalava proprio gli Studi sulla poesia dialettale del Novecento (1983), da me curati e dedicati ad Achille Curcio, come esempio di questo comparativismo consapevole, necessario senz'altro alla letteratura europea contemporanea, attenta ai linguaggi che come detriti nella pasta della comunicazione di oggi 
spostano su un piano di opportunità significativo le occasioni del parlante europeo, le sue chances.

Sicché non ci resta che ricordare che le strategie formali del testo, nella mente del lettore comparatista, non sono separabili dalla doppia natura della lettura che segue un percorso di acquisizioni. Per il lettore che sa comparare codici e linguaggi, dialettalità e superlingue, dunque:

1. il testo esiste nella dimensione del significante fonoritmico, persuasivo e percussivo;

2. il testo convince il lettore dello spazio innovativo che è nel linguaggio della poesia, e della dialettalità come dimensione possibile del pensiero;

3. il testo attraversa gli spazi delle coimplicazioni simboliche e tematiche;

4. il testo porta, per vie del tutto naturali, alla comparazione con la lingua e fra le lingue: un registro della differenza, e un codice a cui il lettore accede anche aprendo varchi nella ricezione dal testo dialettale alla mente italofona o romanza, e viceversa;

5. la poesia viene intesa come codice entro il codice esclusivo di ogni processo creativo del senso, e dunque impone una ennesima comparazione fra norma comunicativa e discorso del testo poetico, come dimostra l'opera dei dialettali italiani contemporanei.

$\mathrm{Al}$ poeta dialettale spetta dunque una responsabilità non marginale nella nostra epoca: a lui la possibilità di ricucire gli strappi della nostra coscienza nel double-bind del comparativismo.

\section{Bibliografia essenziale}

AA.VV. Studi sulla poesia dialettale del Novecento. A cura di L. Tassoni. Catanzaro: Comune di Catanzaro, 1983.

AA.VV. Le parole di legno. Poesia in dialetto del '900 italiano. A cura di M. Chiesa e G. Tesio. Milano: Mondadori, 1984.

Hermann W. Haller. La festa delle lingue. La letteratura dialettale in Italia. Roma: Carocci, 2002.

Claudio GUILLÉN. L’uno e il molteplice. Introduzione alla letteratura comparata. Bologna: Il Mulino, 1993.

Gerhard RoHLFs. Grammatica storica della lingua italiana e dei suoi dialetti. Torino: Einaudi, 1968.

Luigi TASSONI. Senso e discorso nel testo poetico. Roma: Carocci, 1999. 